The Impact of Large-Scale Surveys on Pulsating Star Research ASP Conference Series, Vol. 203, 2000

L. Szabados \& D. W. Kurtz, eds.

\title{
Observational Proof of the ZZ Ceti Red Edge
}

\author{
A. Kanaan ${ }^{1}$, D. E. Winget ${ }^{2}$, S. O. Kepler ${ }^{3}$, M. H. Montgomery ${ }^{4}$
}

With a time-series CCD photometry survey, we have demonstrated clearly that the observed red edge for the ZZ Ceti stars instability strip at $11,000 \mathrm{~K}$ is not an observational selection effect. Previous surveys for variability among hydrogen atmosphere white dwarfs at around $11,000 \mathrm{~K}$ have been carried out using high speed photometry which suffers from variable extinction effects that start becoming important at periods of 15 minutes. In our survey we constantly monitor the sky brightness as well as one or more comparison stars. This is done through the same color filter, therefore minimizing adverse effects of differential extinction. The fact that the theoretical red edge should be around $8,000 \mathrm{~K}$ remains, but effects not included in the theory, especially convection-pulsation interaction, could explain it.

Table 1. Photometric limits on variability for cool DAs

\begin{tabular}{llccc}
\hline WD & Alias & Temperature & Length (h) & Limit (mmag) \\
\hline $0032-175$ & G226-135 & 9980 & 4.8 & $<2$ \\
$0033+016$ & G001-007 & 11184 & 5.5 & $<3$ \\
$0101+048$ & G001-045 & 8750 & 6.7 & $<2$ \\
$0135-052$ & L870-002 & 8700 & 4.5 & $<3$ \\
$0816+387$ & G111-071 & 7710 & 6.6 & $<3$ \\
$0913+442$ & G116-016 & 8750 & 8.9 & $<2$ \\
$0955+247$ & G049-033 & 8600 & 9.2 & $<2$ \\
$1147+255$ & G121-022 & 10317 & 8.0 & $<3$ \\
$1244+149$ & G061-017 & 11068 & 4.8 & $<5$ \\
$1507-105$ & GD176 & 10640 & 4.2 & $<3$ \\
$1537+651$ & GD348 & 9910 & 5.0 & $<2$ \\
$1539-035$ & GD189 & 10457 & 7.1 & $<2$ \\
$1655+215$ & G169-34 & 9578 & 4.5 & $<5$ \\
$1840-111$ & G155-34 & 10389 & 5.0 & $<3$ \\
$1857+119$ & G141-54 & 10182 & 3.5 & $<5$ \\
$2136+229$ & G126-018 & 10652 & 7.5 & $<2$ \\
$2246+223$ & G067-023 & 11131 & 6.1 & $<2$ \\
\hline
\end{tabular}

Table 1 presents the results of our survey. Limit is the upper limit for variability for periods shorter than two hours. Length is the duration of our observations.

\footnotetext{
${ }^{1}$ Departamento de Física, Universidade Federal de Santa Catarina, Brazil

${ }^{2}$ Department of Astronomy, University of Texas at Austin, U.S.A.

${ }^{3}$ Departamento de Astronomia, Universidade Federal do Rio Grande do Sul, Brazil

${ }^{4}$ Institut für Astronomie, Universität Wien, Austria
} 